\title{
A CONTINUATION THEORY FOR WEAKLY INWARD MAPS
}

\author{
by DONAL O'REGAN
}

(Received 3 December, 1996)

\begin{abstract}
Fixed point and random fixed point theorems are presented for weakly inward maps. Also a continuation theorem for weakly inward maps is presented.
\end{abstract}

1. Introduction. This paper presents some new results for weakly inward mappings defined on a Banach space $E$. The paper will be divided into two main sections. In Section 2 we first establish a continuation theorem for weakly inward multivalued maps. This result together with a result of Deimling [2,3] will yield a variety of fixed point results for weakly inward maps in shells of Banach spaces. The results we present would arise naturally if a theory of fixed point index was available in a Banach space setting. However this is not known for general Banach spaces even when the maps are compact; see $[7,15]$ for recent results. The fixed point theory we derive will be useful in studying the existence of nonnegative solutions to positone and non-positone problems; see $[\mathbf{1 0 , 1 6 ]}$ for applications in the single valued case. The case when $E$ is a locally convex Hausdorff linear topological space will also be discussed in Section 2. In Section 3 we present some random fixed point theorems for weakly inward maps. Our paper was motivated by work of Deimling [2,3], Precup [12], Reich [13] and Webb [18].

For the remainder of this section we gather together some definitions and known facts. Let $E$ be a Banach space (or more generally a locally convex Hausdorff linear topological space) and let $C$ be a closed convex subset of $E$. The set

$$
I_{c}(x)=\{x+\lambda(y-x): \lambda \geq 0, y \in C\} \text { for } x \in C
$$

is called the inward set at $x$. A mapping $F: C \rightarrow 2^{E}$ (here $2^{E}$ denotes the family of all nonempty subsets of $E$ ) is said to be weakly inward with respect to $C$ if

$$
F(x) \cap \overline{I_{C}(x)} \neq \emptyset \text { on } C \text {. }
$$

REMARK. It is worth noting that some authors define weakly inward w.r.t. $C$ to be the more restrictive condition $F(x) \subseteq \overline{I_{C}(x)}$ on $C$.

Recall [3] if $C$ is a closed convex subset of a Banach space then

$$
\overline{I_{C}(x)}=\bar{I}_{C}(x)=x+\overline{\{\lambda(y-x): \lambda \geq 1, y \in C\}} \text {. }
$$

Let $X$ and $Y$ be topological spaces. A mapping $F: X \rightarrow 2^{Y}$ is upper semicontinuous (u.s.c.). if the set $F^{-1}(B)=\{x \in X: F(x) \cap B \neq \emptyset\}$ is closed for any closed set $B$ in $Y . F$ is lower semicontinuous (l.s.c.) if $F^{-1}(B)$ is open for any open set $B$ in $Y$. If $F$ is u.s.c. and l.s.c. then we say $F$ is continuous. Now let $E$ be a Banach space and $\Omega_{E}$ the bounded subsets of $E$. The Kuratowski measure of noncompactness is the map $\alpha: \Omega_{E} \rightarrow[0, \infty]$ defined by

$$
\alpha(A)=\inf \left\{\epsilon>0: A \subseteq \bigcup_{i=1}^{n} X_{i} \text { and } \operatorname{diam}\left(X_{i}\right) \leq \epsilon\right\} ; \text { here } A \in \Omega_{E}
$$

Glasgow Math. J. 40 (1998) 311-321. 
Let $Z$ be a nonempty subset of $E$ and $F: Z \rightarrow 2^{E}$. $F$ is called a $k$-set contraction ( $k \geq 0$ a constant) if $\alpha(F(W)) \leq k \alpha(W)$ for all bounded sets $W$ of $Z$. We call $F$ a condensing map if $F$ is a 1-set concentration and $\alpha(F(W)<\alpha(W)$ for all bounded sets $W \subseteq Z$ with $\alpha(W) \neq 0$ (of course $F$ being a 1-set contraction can be removed from the definition of condensing if we wish).

Next we state a result of Deimling [3] which will be used in Section 2.

THEOREM 1.1. Let $E$ be a (real) Banach space and $C$ a closed, bonded, convex subset of $E$. Suppose $F: C \rightarrow 2^{E}$ is a u.s.c. condensing map with closed convex values. If $F(x) \cap \overline{I_{C}(x)} \neq \emptyset$ on $C$ then $F$ has a fixed point in $C$.

Let $(\Omega, \mathcal{A})$ denote a measurable space. For a metric space $(X, d)$ we denote by $C D(X)$ all nonempty closed subsets of $X$. Suppose $Z$ is a nonempty subset of $X$ and $F: Z \rightarrow 2^{X}$. Now $F$ is called hemicompact if each sequence $\left\{\left(x_{n}\right)\right\}_{n=1}^{\infty}$ in $Z$ has a convergent subsequence whenever $d\left(x_{n}, F\left(x_{n}\right)\right) \rightarrow 0$ as $n \rightarrow \infty$; recall if $Y$ is a nonempty subset of $X$ then $d(x, Y)=$ $\inf _{y \in Y} d(x, y)$. A mapping $F: \Omega \rightarrow 2^{X}$ is measurable (respectively weakly measurable) if for every closed (respectively open) subset $D$ of $X, F^{-1}(D)=\{w \in \Omega: F(w) \cap D \neq \emptyset\} \in \mathcal{A}$. A mapping $F: \Omega \times Z \rightarrow 2^{X}$ is called a random operator if for every $x \in Z$ the map $F(., x): \Omega \rightarrow X$ is measurable. A random operator $F: \Omega \times Z \rightarrow 2^{X}$ is called continuous (hemicompact etc.) if for each $w \in \Omega, F(w,$.$) is continuous (hemicompact etc.). The single valued map \phi: \Omega \rightarrow X$ is said to be (i) a deterministic fixed point of $F$ if $\phi(w) \in F(w, \phi(w))$ for all $w \in \Omega$; (ii) a random fixed point of $F$ if $\phi$ is a measurable map such that $\phi(w) \in F(w, \phi(w))$ for all $w \in \Omega$.

We now state a very recent result due to Tan and Yuan [17].

Theorem 1.2. Let $(\Omega, \mathcal{A})$ be a measurable space and $Z$ a nonempty separable complete subset of a metric space $(X, d)$. Suppose the map $F: \Omega \times Z \rightarrow C D(X)$ is a random continuous hemicompact map. Then $F$ has a deterministic fixed point iff $F$ has a random fixed point.

2. Fixed point theory. In this section we establish a continuation type result for weakly inward maps. First we assume $E$ is a Banach space and $C \subseteq E$ is a closed convex subset of $E$. Let $U_{0}$ be a bounded open subset of $E$ and $U=U_{0} \cap C$. For notational purposes let $C K(E)$ denote the family of all nonempty, convex, compact subsets of $E$. Suppose $L: \bar{U} \rightarrow E$ is a continuous operator (single valued). By $I K_{\partial U}(\bar{U}, E ; L)$ we mean the set of all u.s.c. condensing, weakly inward w.r.t. $C$ (i.e. $F(x) \cap \overline{I_{C}(x)} \neq \emptyset$ on $\bar{U}$ ), maps $F: \bar{U} \rightarrow C K(E)$ such that $L-F$ is zero free on $\partial U$ (i.e. $L x \notin F x$ for all $x \in \partial U$ ); here $\partial U$ denotes the boundary of $U$ in $C$. A mapping $F \in I K_{\partial U}(\bar{U}, E ; L)$ is $L$-essential in $I K_{\partial U}(\bar{U}, E ; L)$ if for every $G \in I K_{\partial U}(\bar{U}, E ; L)$ with $\left.F\right|_{\partial U}=\left.G\right|_{\partial U}$ we have that $L-G$ has a zero in $U$. Otherwise $F$ is $L$-inessential in $I K_{\partial U}(\bar{U}, E ; L)$ i.e. there exists $G \in I K_{\partial U}(\bar{U}, E ; L)$ with $\left.F\right|_{\partial U}=\left.G\right|_{\partial U}$ and $L-G$ is zero free on $U$. Two maps $F, G \in I K_{\partial U}(\bar{U}, E ; L)$ are homotopic in $I K_{\partial U}(\bar{U}, E ; L)$ written $F \cong G$ in $I K_{\partial U}(\bar{U}, E ; L)$ if there is a u.s.c. condensing $[9,11]$ map $N: \bar{U} \times[0,1] \rightarrow C K(E)$ such that $N_{t}(u)=N(u, t)$ : $\bar{U} \rightarrow C K(E)$ belongs to $I K_{\partial U}(\bar{U}, E ; L)$ for each $t \in[0,1]$ and $N_{0}=F, N_{1}=G$.

TheOREM 2.1. Let $E, C, U_{0}, U, L$ be as above and $F \in I K_{\partial U}(\bar{U}, E ; L)$. Then the following are equivalent:

(i) $F$ is L-inessential in $I K_{\partial U}(\bar{U}, E ; L)$; on $\bar{U}$

(ii) there is a map $G \in I K_{\partial U}(\bar{U}, E ; L)$ with $F \cong G$ in $I K_{\partial U}(\bar{U}, E ; L)$ and $L-G$ is zero free 
Proof. We first show (i) implies (ii). Let $G \in I K_{\partial U}(\bar{U}, E ; L)$ with $\left.G\right|_{\partial U}=\left.F\right|_{\partial U}$ and $L-G$ is zero free on $U$. Define $H: \bar{U} \times[0,1] \rightarrow C K(E)$ by

$$
H(x, t)=t G(x)+(1-t) F(x) \text { for }(x, t) \in \bar{U} \times[0,1] .
$$

A standard argument (see $[5,9,11]$ shows $H$ is u.s.c. and condensing. Also for each $t \in[0,1], H_{t} \in$ $I K_{\partial U}(\bar{U}, E ; L)$. To see this first notice $L-H_{t}$ is zero free on $\partial U$ for each $t \in[0,1]$ (since $L-G$ is zero free on $\partial U$ and $\left.\left.F\right|_{\partial U}=\left.G\right|_{\partial U}\right)$. In addition for $x \in \bar{U}$ we have $F(x) \cap \overline{I_{C}(x)} \neq \emptyset$ and $G(x) \cap \overline{I_{C}(x)} \neq \emptyset$ and so, since $H_{t}(x)=t G(x)+(1-t) F(x)$, we have

$$
H_{t}(x) \cap \overline{I_{C}(x)} \neq \emptyset
$$

since $\overline{I_{C}(x)}$ is a convex subset of $E$. Thus $F \cong G$ in $I K_{\partial U}(\bar{U}, E ; L)$.

We next show (ii) implies (i). Let $N: \bar{U} \times[0,1] \rightarrow C K(E)$ be an u.s.c. condensing map with $N_{t} \in I K_{\partial U}(\bar{U}, E ; L)$ for each $t \in[0,1]$ and $N_{0}=G, N_{1}=F$. Let

$$
B=\{x \in \bar{U}: L x \in N(x, t) \text { for some } t \in[0,1]\} .
$$

If $B=\emptyset$ then in particular $L-F=L-N_{1}$ has no zeros so $F$ is L-inessential in $I K_{\partial U}(\bar{U}, E ; L)$. It remains to consider the case when $B \neq \emptyset$. A standard argument (see $[5,9,11])$ shows $B$ is closed. In addition since $\partial U \cap B=\emptyset$ there is a continuous function $\mu: \bar{U} \rightarrow[0,1]$ with $\mu(\partial U)=1$ and $\mu(B)=0$. Define

$$
J: \bar{U} \rightarrow C K(E) \text { by } J(x)=N(x, \mu(x)) .
$$

Now $J$ is u.s.c. and condensing. We claim $\left.J\right|_{\partial U}=\left.F\right|_{\partial U}, L-J$ is zero free on $U$ and $J$ is weakly inward w.r.t. $C$. If this is true then $J \in I K_{\partial U}(\bar{U}, E ; L)$ with $L-J$ zero free on $U$ and $\left.J\right|_{\partial U}=\left.F\right|_{\partial U}$. Consequently $F$ is $L$-inessential in $I K_{\partial U}(\bar{U}, E ; L)$ and we are finished.

It remains to prove the claim. $L-J$ is zero free since $0 \in L(x)-J(x)$ means $L(x) \in$ $N(x, \mu(x)$ ) which means $x \in B$ and so $\mu(x)=0$ (i.e. $L(x) \in N(x, 0)$ ), a contradiction since $L-G$ is zero free. If $x \in \partial U$ then $\mu(x)=1$ and so

$$
J(x)=N(x, \mu(x))=N(x, 1)=F(x) .
$$

Finally to show that $J$ is weakly inward w.r.t. $C$ notice that for fixed $s \in[0,1] N_{s}$ is weakly inward w.r.t. $C$ i.e. $N_{s}(x) \cap \overline{I_{C}(x)} \neq \emptyset$ for $x \in \bar{U}$. For fixed $x \in \bar{U}$ let $\mu(x)=s$ so that $J(x)=N_{s}(x)$ and

$$
J(x) \cap \overline{I_{C}(x)}=N_{s}(x) \cap \overline{I_{C}(x)} \neq \emptyset .
$$

We can do this argument for all $x \in \bar{U}$ and so $J$ is weakly inward w.r.t. $C$.

Theorem 2.1. immediately yields a topological transversality theorem for weakly inward maps.

Theorem 2.2. Let $E, C, U_{0}, U, L$ be as above. Suppose $F$ and $G$ are two maps in $I K_{\partial U}(\bar{U}, E ; L)$ such that $F \cong G$ in $I K_{\partial U}(\bar{U}, E ; L)$. Then $F$ is $L$-essential in $I K_{\partial U}(\bar{U}, E ; L)$ iff $G$ is 
$L$-essential in $I K_{\partial U}(\bar{U}, E ; L)$.

Proof. Suppose $F$ is $L$-inessential in $I K_{\partial U}(\bar{U}, E ; L)$. Then Theorem 2.1 guarantees a map $T \in I K_{\partial U}(\bar{U}, E ; L)$ with $F \cong T$ in $I K_{\partial U}(\bar{U}, E ; L)$ and $L-T$ is zero free on $\bar{U}$. Thus $G \cong T$ in $I K_{\partial U}(\bar{U}, E ; L)$ so $G$ is $L$-inessential in $I K_{\partial U}(\bar{U}, E ; L)$ by Theorem 2.1. Symmetry will now imply $F$ is $L$-inessential in $I K_{\partial U}(\bar{U}, E ; L)$ iff $G$ is $L$-inessential in $I K_{\partial U}(\bar{U}, E ; L)$.

For the remainder of this section let $L=I$ (the identity operator).

Theorem 2.3. Let $E, C, U_{0}, U$ be as above, $u_{0} \in U=U_{0} \cap C$. Then the constant map $F: \bar{U} \rightarrow\left\{u_{o}\right\}$ is I-essential in $I K_{\partial U}(\bar{U}, E ; I)$.

Proof: Let $G: \bar{U}: \rightarrow C K(E)$ be any u.s.c. condensing, weakly inward w.r.t. $C$, map with $\left.G\right|_{\partial U}=\left.F\right|_{\partial U}=\left\{u_{0}\right\}$. We must show $G$ has a fixed point in $U$. Since $U_{0}$ is a bounded subset of $E$ we may choose $R>0$ so that

$$
U_{0} \subset\{x \in E:\|x\|<R\} \text { and } G(\bar{U}) \subset\{x \in E:\|x\|<R\} .
$$

Let

$$
D=C \cap\{x \in E:\|x\|<R+1\}
$$

and define

$$
J(x)= \begin{cases}G(x), & x \in \bar{U} \\ \left\{u_{0}\right\}, & x \in \bar{D} / \bar{U} .\end{cases}
$$

Now $J: \bar{D} \rightarrow C K(E)$ is a u.s.c. condensing map. We claim $J$ is weakly inward w.r.t. $\bar{D}$. If this is true then Theorem 1.1 implies that $J$ has a fixed point, say $y$, in $\vec{D}$ (i.e. $y \in J(y)$ ). It is immediate that $y \in U$ so $y \in G(y)$.

It remains to prove the claim. If $x \in \bar{D} / \bar{U}$ then $J(x)=\left\{u_{0}\right\} \in \overline{I_{\bar{D}}(x)}$ since $u_{0} \in U_{0} \cap C$ (so $\left.u_{0} \in \bar{D}\right)$. Now let $x \in \bar{U}$ and take $y \in J(x)=G(x)$ with $y \in \overline{I_{C}(x)}$. Now there exists $\left\{\lambda_{n}\right\}_{n \in \mathbb{N}}$, with $\lambda_{n} \geq 1$ for $n \in \mathbb{N}$ and $\left\{z_{\lambda_{n}}\right\}_{n \in \mathbb{N}} \subseteq C$ with

$$
\left\|y-\left[x+\lambda_{n}\left(z_{\lambda_{n}}-x\right)\right]\right\| \rightarrow 0 \text { as } n \rightarrow \infty .
$$

Let

$$
v_{\lambda_{n}}=x+\lambda_{n}\left(z_{\lambda_{n}}-x\right)
$$

Then $v_{\lambda_{n}} \rightarrow y$ as $n \rightarrow \infty$ and so

$$
v_{\lambda_{n}} \in\{x \in E:\|x\|<R+1\}
$$

for $n \in \mathbb{N}$ sufficiently large. Let $\mu_{n}=\frac{1}{\lambda_{n}}$. Then

$$
z_{\lambda_{n}}=\left(1-\mu_{n}\right) x+\mu_{n} v_{\lambda_{n}}
$$

so $z_{\lambda_{n}} \in\{x \in E:\|x\|<R+1\}$ for $n$ sufficiently large. In addition since $\left\{z_{\lambda_{n}}\right\}_{n \in \mathbb{N}^{c} C}$ we have $z_{\lambda_{n}} \in \bar{D}$ for $n$ sufficiently large, and $\| y-\left(x+\lambda_{n}\left(z_{\lambda_{n}}-x\right) \| \rightarrow 0\right.$ as $n \rightarrow \infty$. Thus $y \in \overline{I_{\bar{D}}(x)}$.

Theorems 2.2. and 2.3 will now be used to present a variety of fixed point results. 
THEOREM 2.4. Let $E$ be a Banach space and $C$ a closed convex subset of $E$. Let $U_{0}$ be a bounded open subset of $E$ and $u_{0} \in C \cap U_{0}=U$. In addition assume

$h: \bar{U} \times[0,1] \rightarrow C K(E)$ is a u.s.c. condensing map such that $h(x, 0)=\left\{u_{0}\right\}$

for all $x \in \bar{U}$ and such that for each $t \in[0,1], h_{t}()=.h(., t): \bar{U} \rightarrow C K(E)$

is weakly inward w.r.t $C$ and $x \notin h_{t}(x)$ for $x \in \partial U$

holds. Then $h(., 1)$ has a fixed point $x \in U$.

Proof. The result follows immediately from Theorem 2.2. and Theorem 2.3 .

THEOREM 2.5. Let $E$ be a Banach space and $C$ a closed convex subset of $E$. Let $U_{0}$ be a bounded open subset of $E, U=U_{0} \cap C$ and $0 \in U$. Suppose $F: \bar{U} \rightarrow C K(E)$ is a u.s.c. I-set contractive, weakly inward w.r.t. $C$, map and $x \notin \lambda F(x)$ for $\lambda \in[0,1]$ and $x \in \partial U$. In addition assume

$$
\begin{aligned}
& \text { if }\left\{x_{n}\right\} \subseteq \bar{U} \text { with } y_{n} \in F\left(x_{n}\right) \text { for all } n \text { and } x_{n}-y_{n} \rightarrow 0 \\
& \text { as } n \rightarrow \infty \text {, then there exists } x \in \bar{U} \text { with } x \in F(x)
\end{aligned}
$$

holds. Then $F$ has a fixed point.

REMARK. If $\bar{U}$ is weakly compact and $I-F: \bar{U} \rightarrow C K(E)$ is demiclosed on $\bar{U}$ then it is easy to see that (2.2) holds. Recall the operator $S: D(S) \subseteq E \rightarrow C K(E)$ is said to be demiclosed on $\mathrm{D}(\mathrm{S})$ if for $x_{n} \in D(S)$ with $y_{n} \in S\left(x_{n}\right)$ for all $n$, the condition $x_{n} \rightarrow x$ and $y_{n} \rightarrow y$ as $n \rightarrow \infty$ imply that $x \in D(S)$ and $y \in S(x)$; here $\rightarrow$ denotes weak convergence.

Proof. For each $n \in\{2,3, \ldots\}$ define

$$
F_{n}=\left(1-\frac{1}{n}\right) F: \bar{U} \rightarrow C K(E) .
$$

Clearly $F_{n}$ is u.s.c. $\left(1-\frac{1}{n}\right)$-set contractive map. In addition for $x \in \bar{U}$ we have $F(x) \cap \overline{I_{C}(x)}$ $\neq \emptyset$ and $0 \in \overline{I_{C}(x)}$ since $0 \in U \subseteq C$. Consequently, since $F_{n}(x)=\left(1-\frac{1}{n}\right) F(x)+\frac{1}{n} 0$, we have

$$
F_{n}(x) \cap \overline{I_{C}(x)} \neq \emptyset
$$

since $\overline{I_{C}(x)}$ is a convex subset of $E$. Thus $F_{n}$ is weakly inward w.r.t. $C$. Let

$$
h(x, t)=t F_{n}(x) \text { for }(x, t) \in \bar{U} \times[0,1] .
$$

It is easy to check that $h$ satisfies (2.1) with $u_{o}=0$. Theorem 2.4 implies that $F_{n}$ has a fixed point $x_{n} \in \bar{U}$. Choose $y_{n} \in F\left(x_{n}\right)$ with $x_{n}=\left(1-\frac{1}{n}\right) y_{n}$. Notice

$$
x_{n}-y_{n}=-\frac{1}{n} y_{n} \rightarrow 0
$$

since $F(\bar{U})$ is bounded. Now apply (2.2). We are of course guaranteed the existence of $x \in \bar{U}$ with $x \in F(x)$. 
Next we establish some fixed point results in shells of Banach spaces.

THEOREM 2.6. Let $E$ be a Banach space and $C$ a closed, convex subset of $E$. Let $W$ and $V$ be open bounded subsets of $E$ and let $U_{1}=W \cap C$ and $U_{2}=V \cap C$. Assume $\overline{U_{1}} \subset U_{2}$ (proper) and $F: \overline{U_{2}} \rightarrow C K(E)$ is a u.s.c. condensing, weakly inward w.r.t. $C$, map. In addition suppose

$$
\begin{gathered}
x \notin F(x) \text { for } x \in \partial U_{1} \cup \partial U_{2}, \\
F: \overline{U_{1}} \rightarrow C K(E) \text { is I-inessential in } I K_{\partial U_{1}}\left(\overline{U_{1}}, E ; I\right)\left(\text { i.e. }\left.F\right|_{\overline{U_{1}}} \text { is I-inessential }\right)
\end{gathered}
$$

and

$$
F: \overline{U_{2}} \rightarrow C K(E) \text { is I-essential in } I K_{\partial U_{2}}\left(\overline{U_{2}}, E ; I\right)
$$

hold. Then $F$ has at least one fixed point in $U_{2} / \overline{U_{1}}$.

Proof. Suppose $F$ has no fixed points in $U_{2} / \overline{U_{1}}$. Now (2.4) implies that there exists a u.s.c. condensing, weakly inward w.r.t. $C$, map $\theta: \overline{U_{1}} \rightarrow C K(E)$ with $\left.\theta\right|_{\partial U_{1}}=\left.F\right|_{\partial U_{1}}$ and $x \notin \theta(x)$ for $x \in \overline{U_{1}}$. Define $\Phi: \overline{U_{2}} \rightarrow C K(E)$ by

$$
\Phi(x)= \begin{cases}F(x), & x \in \overline{U_{2}} / \overline{U_{1}} \\ \theta(x), & x \in \overline{U_{1}} .\end{cases}
$$

Now $\Phi$ is a u.s.c. condensing, weakly inward w.r.t. $C$, map. Also $\Phi$ has no fixed points on $\overline{U_{2}}$ (since $\theta$ has no fixed points on $\overline{U_{1}}$ and $F$ has no fixed points on $U_{2} / \overline{U_{1}}$ ). This of course contradicts (2.5).

Our next theorem was motivated by results of Precup[12].

THEOREM 2.7. Let $E$ be a Banach space and $C$ a closed, convex subset of E. Let $W$ and $V$ be open bounded subsets of $E$ and let $U_{1}=W \cap C, U_{2}=V \cap C$ and $\overline{U_{1}} \subset U_{2}$ (proper) with $0 \in U_{1}$. In addition assume

$N: \overline{U_{2}} \times[0,1] \rightarrow C K(E)$ is a u.s.c. condensing map such that for each $t \in[0,1]$,

$N_{t}()=.N(., t): \overline{U_{2}} \rightarrow C K(E)$ is weakly inward w.r.t. $C$, with $N(x, 0)=\{0\}$

for all $x \in \overline{U_{2}}$ and for each $t \in[0,1]$ we have $x \notin N_{t}(x)$

for all $x \in \partial U_{2}$;

$H: \overline{U_{1}} \times[0,1] \rightarrow C K(E)$ is a u.s.c. condensing map such that for each $t \in[0,1]$,

$H_{t}()=.H(., t): \overline{U_{1}} \rightarrow C K(E)$ is weakly inward w.r.t. $C$, and for each

$t \in[0,1]$ we have $x \notin H_{i}(x)$ for all $x \in \partial U_{1}$;

$$
H(x, 1)=N(x, 1) \text { for all } x \in \overline{U_{1}}
$$

and

$$
x \notin H(x, 0) \text { for all } x \in U_{1} .
$$

Then $N(., 1)$ has a fixed point in $U_{2} / \overline{U_{1}}$.

Proof. From Theorem 2.3 we know that the zero map is $I$-essential in $I K_{\partial U_{2}}\left(\overline{U_{2}}, E ; I\right)$. Then (2.6) together with Theorem 2.2 implies 


$$
N(., 1): \overline{U_{2}} \rightarrow C K(E) \text { is } I \text {-essential in } I K_{\partial U_{2}}\left(\overline{U_{2}}, E ; I\right) \text {. }
$$

Now (2.9) (and also (2.7)) implies that $H(x, 0)$ is $I$-inessential in $I K_{\partial U_{1}}\left(\overline{U_{1}}, E ; I\right)$ (this is immediate from the definition of $I$-inessential in $I K_{\partial U_{1}}\left(\overline{U_{1}}, E ; I\right)$. This together with (2.7), (2.8) and Theorem 2.2 yields

$$
N(., 1)=H(., 1): \overline{U_{1}} \rightarrow C K(E) \text { is } I \text {-inessential in } I K_{\partial U_{1}}\left(\overline{U_{1}}, E ; I\right) .
$$

Now (2.10), (2.11) (see also (2.6), (2.7) and (2.8)) imply that (2.3), (2.4) and (2.5) hold. The result now follows from Theorem 2.6.

TheOREM 2.8. Let $E$ be a Banach space and $C$ a closed, convex subset of $E$. Let $W$ and $V$ be open bounded subsets of $E$ and let $U_{1}=W \cap C, U_{2}=V \cap C$ and $\overline{U_{1}} \subset U_{2}$ (proper). Suppose $0 \in U_{1}$ and $F: \overline{U_{2}} \rightarrow C K(E)$ is a u.s.c. condensing, weakly inward w.r.t.C, map. In addition assume

$$
x \notin \lambda F(x) \text { for } \lambda \in[0,1] \text { and } x \in \partial U_{2},
$$

$$
\text { there exists } a v \in C /\{0\} \text { with } x \notin F(x)+\delta v \text { for } \delta \geq 0 \text { and } x \in \partial U_{1}
$$

and

$$
F(.)+\mu v: \overline{U_{1}} \rightarrow C K(E) \text { is weakly inward w.r.t. } C \text { for all } \mu \geq 0
$$

hold. Then $F$ has a fixed point in $\overline{U_{2}} / U_{1}$.

REMARK. If $F$ is single valued and $C$ is a cone then (2.14) holds. To see this recall [15] that $F: \overline{U_{1}} \rightarrow C K(E)$ weakly inward w.r.t. $C$ (in this setting) means

$$
x \in \partial_{E} C \cap U_{1}, x^{*} \in C^{*}, x^{*}(x)=0 \text { implies } x^{*}(F(x)) \geq 0
$$

here $C^{*}=\left\{x^{*} \in E^{*}: x^{*}(y) \geq 0\right.$ for all $\left.y \in C\right\}$. Now if $x \in \partial_{E} C \cap U_{1}, x^{*} \in C^{*}, x^{*}(x)=0$ then

$$
x^{*}(F(x)+\mu v)=x^{*}(F(x))+\mu x^{*}(v) \geq 0,
$$

since $v \in C$ and $x^{*} \in C^{*}$. Thus (2.14) holds in this case. In fact it is also easy to see that (2.14) is true even if $F$ is multivalued and $C$ is a cone. In this case $\overline{I_{C}(x)}$ is a wedge.

Proof. Now $x \notin F(x)$ for $x \in \partial U_{2} \cup \partial U_{1}$ (see (2.12) with $\lambda=1$ and (2.13) with $\delta=0$ ). Choose $M>0$ such that $\|\{y: y \in F(x)\}\| \leq M$ for all $x \in \overline{U_{1}}$ and choose $\delta_{0}>0$ such that

Now let

$$
\left\|\delta_{0} v\right\|>M+\sup \left\{\|x\|: x \in \overline{U_{1}}\right\} .
$$

$$
N(., t)=t F(.) \text { and } H(., t)=F(.)+(1-t) \delta_{o} v \text {. }
$$

Clearly (2.12), (2.13) (with $\left.\delta=(1-t) \delta_{0}\right)$, and (2.14) imply that (2.6) and (2.7) in Theorem 2.7 are satisfied. In addition $(2.8)$ is true since $N(x, 1)=F(x)=H(x, 1)$ for $x \in \overline{U_{1}}$ and finally (2.15) implies (2.9) is satisfied. The result now follows from Theorem 2.7.

REMARK. Let $W I K(\bar{U}, E)$ denote the family of all u.s.c. condensing maps $F: \bar{U} \rightarrow 2^{E}$ with nonempty, compact, $R_{\delta}$ (or more generally acyclic $[4,5]$ ) values and with $F(x) \subseteq \overline{I_{C}(x)}$ for 
$x \in \bar{U}$. In the usual way we can define $W I K_{\partial U}(\bar{U}, E ; L)$ and $L$-essential in $W I K_{\partial U}(\bar{U}, E ; L)$. Exactly the same reasoning as in this section establishes an analogue of Theorem 2.2 and Theorem 2.6 (for maps in $W I K_{\partial U}(\bar{U}, E ; L)$ ). We also obtain the analogue of Theorems 2.32.5, 2.7-2.8 once we produce the analogue of Therorem 1.1 in this setting. This result (with extra conditions) can be found in Fitzpatrick and Petryshyn [4, Corollary 1 and Theorem 4].

The results in this section could be extended also to the situation when $E, d$ is a locally convex metrizable linear topological space. For convenience we take $L=I$ (for the more general case follow the ideas below and the ideas in [9]). Let $X$ be a subset of $E$. A multivalued bounded map $F: X \rightarrow C K(E)$ is said to be a $P$-concentrative mapping [8] if for each $p \in P(P$ is a defining system of seminorms on $E)$ and each bounded non-p-precompact subset $M$ of $X$ (i.e. $M$ is not precompact in the pseudonormed space $(E, p)$ ) we have

$$
\alpha_{p}(F(M))<\alpha_{p}(M),
$$

where $\alpha_{p}($.$) denotes the measure of noncompactness [8] in the pseudonormed space (E,p).$

Let $C$ be a complete convex subset of $E, U_{0}$ a $d$-bounded open subset of $E$ and $U=U_{0} \cap C$. $P I K_{\partial U}(\vec{U}, E ; I)$ denotes the set of all u.s.c. $P$-concentrative, weakly inward w.r.t. $C$, maps $F: \bar{U} \rightarrow C K(E)$ which are fixed point free on $\partial U$. A mapping $F \in P I K_{\partial U}(\bar{U}, E ; I)$ is $I$-essential in $P I K_{\partial U}(\bar{U}, E ; I)$ if every $G \in P I K_{\partial U}(\bar{U}, E ; I)$ with $\left.F\right|_{\partial U}=\left.G\right|_{\partial U}$ has a fixed point in $U$. Otherwise $F$ is $I$-inessential in $P I K_{\partial U}(\bar{U}, E ; I)$. Two maps $F, G \in P I K_{\partial U}(\bar{U}, E ; I)$ are homotopic in $P I K_{\partial U}(\bar{U}, E ; I)$ written $F \cong G$ in $P I K_{\partial U}(\bar{U}, E ; l)$ if there is a u.s.c. $P$-concentrative map $N: \bar{U} \times[0,1] \rightarrow C K(E)$ such that $N_{t}(u)=N(u, t): \bar{U} \rightarrow C K(E)$ belongs to $P I K_{\partial U}(\bar{U}, E ; I)$ for each $t \in[0,1]$ and $N_{0}=F, N_{1}=G$.

The ideas used to prove Theorem 2.1 and Theorem 2.2 together with those in [11] immediately yields the following analogue of Theorem 2.2 .

TheOrem 2.9. Let $E, C, U_{0}, U$ be as above. Suppose $F$ and $G$ are two maps in $P I K_{\partial U}(\bar{U}, E ; I)$ such that $F \cong G$ in $P I K_{\partial U}(\bar{U}, E ; I)$. Then $F$ is I-essential in $P I K_{\partial U}(\bar{U}, E ; I)$ iff $G$ is I-essential in $P I K_{\partial U}(\bar{U}, E ; I)$.

This immediately produces an analogue of Theorem 2.6.

THEOREM 2.10. Let $E$ be a locally convex metrizable linear topological space and $C a$ complete, convex subset of $E$. Let $W$ and $V$ be open d-bounded subsets of $E$ and let $U_{1}=W \cap C$ and $U_{2}=V \cap C$. Assume $\overline{U_{1}} \subset U_{2}$ (proper) and $F: \overline{U_{2}} \rightarrow C K(E)$ is a u.s.c. P-concentrative, weakly inward w.r.t. C, map. In addition suppose

$$
\begin{gathered}
x \notin F(x) \text { for } x \in \partial U_{1} \cup \partial U_{2}, \\
F: \overline{U_{1}} \rightarrow C K(E) \text { is I-inessential in } P I K_{\partial U_{1}}(\bar{U}, E ; I)
\end{gathered}
$$

and

$$
F: \overline{U_{2}} \rightarrow C K(E) \text { is I-essential in } P I K_{\partial U_{2}}\left(\overline{U_{2}}, E ; I\right)
$$

hold. Then $F$ has at least one fixed point in $U_{2} / \overline{U_{1}}$.

We can also obtain analogues of Theorems $2.3-2.5,2.7-2.8$ once we produce an analogue of Theorem 1.1. An analogue of Theorem 1.1. in the locally convex Hausdorff linear topological space setting (with some extra conditions) may be found in Reich $[13,14]$. 
We conclude this section by discussing briefly 1 -set contractive weakly inward maps. Is the property of having a fixed point invariant by homotopy for 1-set contractive maps? It is well known that the answer in general is no. The following discussion indicates (in my opinion) where the difficulties lie. In this setting let $E, C, U, U_{0}$ be as in the beginning of Section 2 . We will consider maps $F: \bar{U} \rightarrow C K(E)$ which satisfy some property, which we will call property (P). $M K_{\partial U}(\bar{U}, E ; I)$ denotes the set of all u.s.c. l-set contractive, weakly inward w.r.t. $C$, maps $F: \bar{U} \rightarrow C K(E)$ such that $F$ is fixed point free on $\partial U$ and $F$ satisfied property (P). A mapping $F \in M K_{\partial U}(\bar{U}, E ; I)$ is $I$-essential in $M K_{\partial U}(\bar{U}, E ; I)$ if every $G \in M K_{\partial U},(\bar{U}, E ; I)$ with $\left.F\right|_{\partial U}=\left.G\right|_{\partial U}$ has a fixed point in $U$. Two maps $F, G \in M K_{\partial U}(\bar{U}, E ; I)$ are homotopic in $M K_{\partial U}(\bar{U}, E ; I)$ written $F \cong G$ in $M K_{\partial U}(\bar{U}, E ; I)$ if there is a u.s.c. 1-set contractive map $N: \bar{U} \times[0,1] \rightarrow C K(E)$ such that $N_{t}(u)=N(u, t): \bar{U} \rightarrow C K(E)$ belongs to $M K_{\partial U}(\bar{U}, E ; I)$ for each $t \in[0,1]$ and $N_{0}=F, N_{1}=G$.

To prove a topological transversality theorem in this setting we need to assume property (P) satisfies the following assumptions:

if $F: \bar{U} \rightarrow C K(E), G: \bar{U} \rightarrow C K(E)$ are two maps which satisfy property (P),

then $\alpha F+(1-\alpha) G: \bar{U} \rightarrow C K(E)$ must satisfy property (P);

here $0<\alpha<1$ is a constant

and

if $\mu: \bar{U} \rightarrow[0,1]$ is a continuous map and $N: \bar{U} \times[0,1] \rightarrow C K(E)$

is a u.s.c. 1-set contractive map with $N_{t} \in M K_{\partial U}(\bar{U}, E ; I)$ for each

$t \in[0,1]$, then the map $J: \bar{U} \rightarrow C K(E)$ given by

$J(x)=N(x, \mu(x))$ must satisfy property $(\mathrm{P})$.

REMARK. Suppose property (P) means the map is condensing, then clearly (2.19) and (2.20) hold.

Essentially the same reasoning as in Theorem 2.1 and Theorem 2.2 yields the following result.

Theorem 2.11. Let $E, C, U_{0}, U$ be as in the beginning of Section 2. Suppose $F$ and $G$ are two maps in $M K_{\partial U}(\bar{U}, E ; I)$ such that $F \cong G$ in $M K_{\partial U}(\bar{U}, E ; I)$. Also assume (2.19) and (2.20) hold. Then $F$ is I-essential in $M K_{\partial U}(\bar{U}, E ; I)$ iff $G$ is I-essential in $M K_{\partial U}(\bar{U}, E ; I)$.

Next we establish an analogue of Theorem 2.3 .

Theorem 2.12. Let E,C, $U_{0}, U$ be as in the beginning of Section 2 and $u_{0} \in U=U_{0} \cap C$. Let $M K_{\partial U}(\bar{U}, E ; I)$ be as described above and assume (2.19) and (2.20) hold. In addition suppose

for any $G \in M K_{\partial U}(\bar{U}, E ; I)$ with $\left.G\right|_{\partial U}=\left\{u_{0}\right\}$ and any $\left\{x_{n}\right\} \subseteq \widetilde{U}$

with $x_{n} \in\left(1-\frac{1}{n}\right) G\left(x_{n}\right)+\frac{1}{n}\left\{u_{0}\right\}$, then there exists $x \in \bar{U}$ with $x \in G(x)$

is satisfied. Then the constant map $F: \bar{U} \rightarrow\left\{u_{0}\right\}$ is I-essential in $M K_{\partial U}(\bar{U}, E ; I)$. 
Proof. Let $G: \bar{U} \rightarrow C K(E)$ be any u.s.c. 1-set contractive, weakly inward w.r.t. $C$, map with $\left.G\right|_{\partial U}=\left.F\right|_{\partial U}=\left\{u_{0}\right\}$ and suppose $G$ satisfies property (P). We must show $G$ has a fixed point in $U$. Let $D$ and $J$ be as in Theorem 2.3. For each $n \in\{2,3 \ldots\}$ consider

$$
J_{n}=\left(1-\frac{1}{n}\right) J+\frac{1}{n} u_{0}: \bar{D} \rightarrow C K(E) .
$$

Clearly $J_{n}$ is a u.s.c. $\left(1-\frac{1}{n}\right)$-set contractive, weakly inward w.r.t. $\bar{D}$ (see the ideas in Theorem 2.3 and Theorem 2.5), map. Now Theorem 1.1 implies $J_{n}$ has a fixed point $x_{n} \in \bar{D}$ for each $n \in\{2,3, \ldots\}$. In fact $x_{n} \in \bar{U}$ so $J\left(x_{n}\right)=G\left(x_{n}\right)$ i.e.

$$
x_{n} \in\left(1-\frac{1}{n}\right) G\left(x_{n}\right)+\frac{1}{n}\left\{u_{0}\right\} .
$$

Now (2.21) implies there exists $x \in \bar{U}$ with $x \in G(x)$. It is immediate that $x \in U$.

Analogues (with extra assumptions) of Theorems 2.4, 2.6-2.8 could also be obtained in this setting.

3. Random fixed point theory. We can use a recent result of Tan and Yuan (see Theorem 1.2) to establish random analogues of the results in Section 2. For completeness we state and prove the random analogue of Theorem 2.8 (this extends results in [1]).

Theorem 3.1 Let $((\Omega, \mathcal{A})$ be a measurable space and $E$ a separable Banach space. Let $C$ be a closed convex subset of $E, W$ and $V$ are open bounded subsets of $E$ with $U_{1}=W \cap C$, $U_{2}=V \cap C, \overline{U_{1}} \subset U_{2}$ (proper) and $0 \in U_{1}$. Suppose $F: \Omega \times \overline{U_{2}} \rightarrow C K(E)$ is a random continuous, condensing, weakly inward w.r.t. $C$ (for each $w \in \Omega, F(w,):. \overline{U_{2}} \rightarrow C K(E)$ is weakly inward w.r.t. C), map and in addition

$$
\begin{gathered}
\text { for each } w \in \Omega, x \notin \lambda F(w, x) \text { for } \lambda \in[0,1] \text { and } x \in \partial U_{2} \\
\text { there exists a } v \in C /\{0\} \text { such that for each } w \in \Omega \\
x \notin F(w, x)+\delta v \text { for } \delta \geq 0 \text { and } x \in \partial U_{1}
\end{gathered}
$$

and

$$
\begin{aligned}
& \text { for each } w \in \Omega, F(w, .)+\mu v: \overline{U_{1}} \rightarrow C K(E) \text { is weakly } \\
& \text { inward w.r.t. } C \text { for all } \mu \geq 0
\end{aligned}
$$

hold. Then $F$ has a random fixed point $x_{0}$ with $x_{0}(w) \in \overline{U_{2}} / U_{1}$ with $x_{0}(w) \in \overline{U_{2}} / U_{1}$ for each $w \in \Omega$.

REMARK. If $F$ is multivalued and $C$ is a cone then (3.3) is satisfied.

Proof. Firstly note $F: \Omega \times \overline{U_{2}} \rightarrow C K(E)$ is hemicompact (see [17, Lemma 2.1]). Also Theorem 2.8 implies that $F$ has a deterministic fixed point. Apply Theorem 1.2 and we deduce that $F$ has a random fixed point.

REMARK. It is also easy to establish a random analogue of Theorem 2.6. We leave this to the reader. 


\section{REFERENCES}

1. I. Beg and N. Shahzad, Random fixed points of weakly inward operators in conical shells, J. Applied Mathematics and Stochastic Analysis 8 (1995), 261-264.

2. K. Deimling, Fixed points of weakly inward maps, Nonlinear Anal., 10 (1986), 1261-1262.

3. K. Deimling, Multivalued Differential Equations (Walter de Gruyter, Berlin, 1992).

4. P. M. Fitzpatrick and W. V. Petryshyn, Fixed point theorems for multivalued noncompact acyclic mappings, Pacific J. Math. 54 (1974), 17-23.

5. L. Gorniewicz, A. Granas and W. Kryszewski, Sur la méthode de l'homotopie dans la théorie des points fixes pour les applications multivoques (Partie 1: Transversalite topologique), C. R. Acid. Sci. Paris, Ser. 1, 307 (1988), 489-492.

6. G. B. Gustafson and K. Schmitt, Nonzero solutions of boundary value problems for second order ordinary and delay differential equations, J. Differential Equations 12 (1972), 129-147.

7. K. Q. Lan and J. R. L. Webb, A fixed point index for weakly inward A-proper maps, Nonlinear Anal. 28 (1997), 315-325.

8. D. O'Regan, Some fixed point theorems for concentrative mappings between locally convex linear topological spaces, Nonlinear Anal., 27 (1996) 1437-1446.

9. D. O'Regan, Coincidence principles and fixed point theory for mappings in locally convex spaces. Rocky Mountain J. Math., to appear.

10. D. O'Regan, Existence of nonnegative solutions to superlinear non-positone problems via a fixed point theorem in cones of Banach spaces, Dynamics of Continuous, Discrete and Impulsive Systems, 3 (1997), 517-530.

11. D. O'Regan, Fixed points for set valued mappings in locally convex linear topological spaces, Mathematical and Computer Modelling, 28(1) (1998), 45-55.

12. R. Precup, On some fixed point theorems of Deimling, Nonlinear Anal. 23 (1994), 1315-1320.

13. S. Reich, Fixed points in locally convex spaces, Math. Z. 125 (1972), 17-31.

14. S. Reich, A fixed point theorem for Frechet spaces, J. Math. Anal. Appl. 78 (1980), 33-35.

15. Shouchuan $\mathrm{Hu}$ and Yong Sun, Fixed point index for weakly inward maps, J. Math. Anal. Appl. 172 (1993), 266-273.

16. Alice Chaljub-Simon and P. Volkmann, Existence of ground states with exponential decay for semi-linear elliptic equations in $\mathbf{R}^{n}, J$. Differential Equations, 76 (1988), 374-390.

17. K. K. Tan and X. Z. Yuan, Random fixed point theorems and approximation in cones, $J$. Math. Anal. Appl, 185 (1994), 378-390.

18. J. R. L. Webb, $A$-properness and fixed points of weakly inward mappings, $J$. London Math. Soc., 27 (1983), 141-149.

\section{Department of Mathematics}

NATIONAL UNIVERSITY OF IRELAND

GALWAY

IRELAND 